\title{
Ballistic Phonon Thermal Transport in Multiwalled Carbon Nanotubes
}

\author{
H.-Y. Chiu, ${ }^{1}$ V. V. Deshpande, ${ }^{1}$ H. W. Ch. Postma, ${ }^{1}$ C. N. Lau, ${ }^{2}$ C. Mikó, ${ }^{3}$ L. Forró,${ }^{3}$ and M. Bockrath ${ }^{1, *}$ \\ ${ }^{1}$ Department of Applied Physics, California Institute of Technology, Pasadena, California 91125, USA \\ ${ }^{2}$ Department of Physics, University of California, Riverside, California 92521, USA \\ ${ }^{3} I P M C / S B$, École Polytechnique Fédérale de Lausanne, CH-1015 Lausanne-EPFL, Switzerland
}

(Received 6 July 2005; published 21 November 2005)

\begin{abstract}
We report electrical transport experiments, using the phenomenon of electrical breakdown to perform thermometry, that probe the thermal properties of individual multiwalled carbon nanotubes. Our results show that nanotubes can readily conduct heat by ballistic phonon propagation. We determine the thermal conductance quantum, the ultimate limit to thermal conductance for a single phonon channel, and find good agreement with theoretical calculations. Moreover, our results suggest a breakdown mechanism of thermally activated $\mathrm{C}-\mathrm{C}$ bond breaking coupled with the electrical stress of carrying $\sim 10^{12} \mathrm{~A} / \mathrm{m}^{2}$. We also demonstrate a current-driven self-heating technique to improve the conductance of nanotube devices dramatically.
\end{abstract}

DOI: 10.1103/PhysRevLett.95.226101

The ultimate thermal conductance attainable by any conductor below its Debye temperature is determined by the thermal conductance quantum $[1,2]$. In practice, phonon scattering reduces the thermal conductivity, making it difficult to observe quantum thermal phenomena except at ultralow temperatures [3]. Carbon nanotubes have remarkable thermal properties [4-7], including conductivity as high as $\sim 3000 \mathrm{~W} / \mathrm{m} \mathrm{K}$ [8]. Here we report the observation of ballistic phonon motion and quantum thermal transport in micron-scale individual carbon nanotube devices, demonstrating the universal limit to thermal transport. In this qualitatively different regime, quantum mechanics limits the entropy flow, giving a maximum thermal conductance and an absolute physical limit to the information bandwidth that a nanotube can transport per unit power $[1,2]$. From our data, we obtain a measurement of the thermal conductance quantum that is in good agreement with theory.

Very recently the thermal conductance of a carbon nanotube attached to an atomic force microscope (AFM) tip has been observed to be independent of the AFM retraction length, and the result was interpreted in terms of ballistic phonon propagation [9]. However, the phonon scattering length and thermal conductance quantum were not determined. In our experiments, we heat multiwalled nanotubes (MWNTs) with an electrical current and monitor temperature by the electrical breakdown phenomenon $[10,11]$. Our data yield an experimental measurement of the thermal conductance quantum, which agrees with theoretical predictions $[1,2,12]$ as well as thermal transport results on cryogenically cooled $\mathrm{Si}_{3} \mathrm{~N}_{4}$ nanobridges [3]. This demonstrates that fundamental knowledge about thermal transport in nanotubes can be obtained from an electrical transport experiment. This knowledge, which is challenging to obtain by other means, also contributes toward understanding thermal management issues relevant to the rational design of nanotube interconnects and logic devices.
PACS numbers: 81.07.De, 63.22.+m, 65.80.+n, 73.63. $-\mathrm{b}$

Fabrication of freestanding and substrate-supported MWNT devices was described elsewhere (e.g., Refs. [13,14]). A device diagram is shown in Fig. 1(a) and a scanning electron microscope (SEM) device image in Fig. 1(b). We mainly studied devices in which electrical leads were placed over the tubes. In the work of Collins et al. [10], it was shown that sufficiently high electrical power dissipation in MWNTs causes the current $I$ to drop in abrupt events separated by $\sim 1 \mathrm{~s}$, due to the ablation of individual nanotube shells, behavior recently imaged by transmission electron microscopy (TEM) [15]. It was carefully argued that the breakdown temperature $T_{B}$ was $\sim 900 \mathrm{~K}$ [10]. Nevertheless, Joule heating alone is not likely to account entirely for the shell breakdown [16].

To address the role of $T_{B}$ in nanotube breakdown, we compared the behavior of both freestanding and supported nanotube devices. In the latter, the substrate yields an additional cooling pathway. Figure 1(c) shows currentvoltage $(I V)$ data from three freestanding nanotube devices with radius $R=10 \mathrm{~nm}$, determined by SEM imaging [17].

Figure 1(d) shows similar data for supported nanotubes, with $R=8 \mathrm{~nm}, 9 \mathrm{~nm}$, and $14 \mathrm{~nm}$. At breakdown, the resistance is directly proportional to the nanotube length, indicating negligible contact resistance. From our $I V$ data, we deduce the breakdown power $P$ for each first shell.

Figure 1(e) shows a log-log plot of $P$ vs $L$. Freestanding tubes approximately follow $P \propto L^{-1}$ behavior, while the supported tubes exhibit $P \propto L$ behavior. This behavior can be understood using a diffusive thermal transport model. For freestanding tubes, the power $P$ to increase the temperature at the tube center by $\Delta T$ is $P=8 \pi R^{2} \kappa \Delta T / L$, where $\kappa$ is the characteristic nanotube thermal conductivity. With $T_{B} \sim 900 \mathrm{~K}$, a linear fit to our data with $\Delta T=$ $600 \mathrm{~K}$ [dashed line in Fig. 1(e)] yields a thermal conductivity of $\kappa \approx 600 \mathrm{~W} / \mathrm{m} \mathrm{K}$, consistent with previous thermal conductivity measurements on individual MWNTs in the diffusive regime [8]. For supported nanotubes, the relation $P \propto L$ indicates that the cooling occurs mainly 

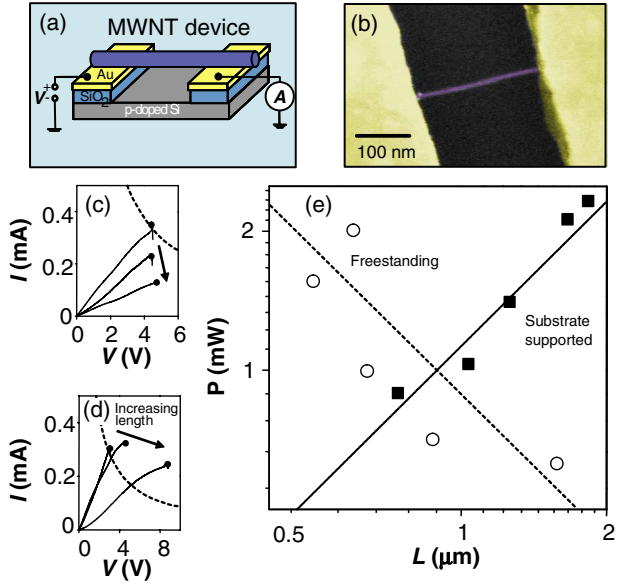

FIG. 1 (color online). (a) Diagram of a freestanding multiwalled nanotube device. (b) SEM image completed device. (c) $I V$ characteristics from freestanding nanotube devices with $R=10 \mathrm{~nm}$. The arrow indicates increasing lengths $(0.50,0.64$, and $1.58 \mu \mathrm{m}$ ). Dotted line: isopower curve. (d) $I V$ characteristic of substrate-supported devices with $R=8,9$, and $14 \mathrm{~nm}$ increasing in length $(0.74,1.26$, and $1.66 \mu \mathrm{m})$ following the arrow. Dotted line: isopower curve. (e) $P$ vs $L$ on a log-log scale for freestanding tubes (open circles) and substrate-supported nanotubes (filled squares). Dotted line: $P \propto L$, dashed line: $P \propto L^{-1}$.

by heat conduction into the substrate. We estimate heat transport in this geometry as between concentric cylinders. This yields $P=2 \pi L \kappa_{s} \Delta T / \ln \left(R_{0} / R\right)$, with $R_{0}$ the outer cylinder radius at which $T$ drops to the ambient value, and $\kappa_{s}$ the substrate thermal conductivity. Taking $R_{0}=50 \mathrm{~nm}$ and $R=10 \mathrm{~nm}$, the fit shown by the dotted line in Fig. 1(e) yields $\kappa_{s} \sim 0.5 \mathrm{~W} / \mathrm{m} \mathrm{K}$, in agreement with the bulk thermal conductivity of $\mathrm{SiO}_{2}, \kappa_{s} \approx 1.5 \mathrm{~W} / \mathrm{m} \mathrm{K}$. Considering the two cases together, our data and analysis indicate that the shell ablation occurs at a well-defined temperature $T_{B}$.

We now focus on freestanding devices, representing a broad range of $L$ and $R$ values. Figure 2 shows $P / 8 \pi R^{2} v s$ $L^{-1}$ for $\sim 30$ samples. Based on diffusive heat transport, we expect plotting the normalized power $P_{N}=P / 8 \pi R^{2} v s$ $L^{-1}$ should yield a straight line with a slope of $T_{B} \kappa$. Remarkably, although the initial trend for the longer tubes appears linear, for nanotubes with $L^{-1} \gtrsim$ $(0.5 \mu \mathrm{m})^{-1}$ (filled squares) $P_{N}$ saturates and becomes $L$ independent [18]. This shows that heat flow from the nanotube occurs at an $L$-independent rate, depending only on $R$. The rest of the shells are then broken, producing a gap in the nanotube. The Fig. 2 inset shows the gap position, normalized to the suspended tube length. This breakpoint is near the center for tubes longer than $\sim 0.5 \mu \mathrm{m}$, but for $L$ in the saturation regime the scatter in the breakpoint values increases. As the statistical distribution of breakpoints reflects the temperature distribution along the nanotube, this suggests the nanotube temperature becomes more spatially uniform as they become shorter than $\sim 0.5 \mu \mathrm{m}$.

Figure 3 shows $P$ vs $R$ on a $\log -\log$ scale. Data from short tube samples follow the power-law fit, showing that
$P \propto R^{\alpha}$, with $\alpha=2.1$. Some data from longer samples, fall near the line, but for the longest nanotubes, the data points fall below the line. The curve followed by the short nanotube data represents an upper limit to $P$; modulo experimental scatter, data for each nanotube fall on or below the curve and achieves the maximum only for nanotubes with $L \lesssim 0.5 \mu \mathrm{m}$. The Fig. 3 inset shows the same data and fit on a linear scale.

We now consider interpretations for this behavior. One possibility is that a dominant metal-nanotube thermal contact resistance $\kappa_{C}$ produces the saturation seen in Fig. 2. This is unlikely, however, as $\kappa_{C}$ was determined to be negligible in Ref. [8], which also used metal contacts as thermal reservoirs, as well as in Ref. [9] which used graphite contacts as a thermal reservoir. Finally, based on the supported tubes' behavior, we would expect $\kappa_{C}$, and hence $P$, to be approximately independent of $R$. The observed systematic relationship $P \propto R^{2}$ differs sharply from this expectation.

Another possibility is that because of ballistic electron transport [19], the electrical current primarily heats the electrodes. In this case, the required power to reach $T_{B}$ may be relatively $L$ insensitive. However, both experiments and theory [20,21] indicate the electronic meanfree path due to phonon emission at the high biases applied to our samples is $\sim 10 \mathrm{~nm}$. Since each optical or zone boundary phonon emission is associated with an energy $\sim 180 \mathrm{meV}$ [21], we expect even for our shortest nanotube studied $(\sim 150 \mathrm{~nm})$ most of the energy eV provided by the electric field to each electron is converted into phonons within the nanotube.

We now discuss the possibility of ballistic phonon transport within the nanotube. In this picture, a diffusive heat transport regime with umklapp interphonon scattering as the dominant scattering mechanism [8] makes a transition to a ballistic center-of-mass motion regime for sample lengths $L \sim 0.5 \mu \mathrm{m}$. This suggests the temperature distribution along the tube should broaden as $L$ decreases, consistent with the data in the Fig. 2 inset. Furthermore, because the characteristic distance the phonons travel be-

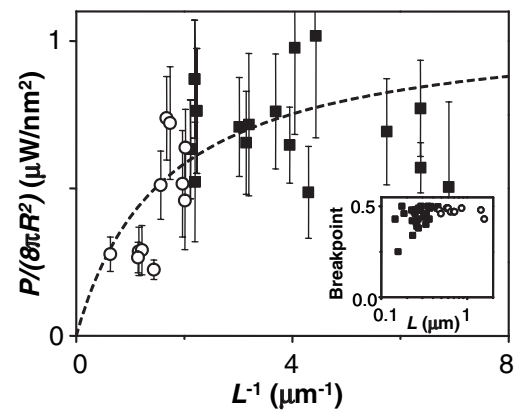

FIG. 2. $P /\left(8 \pi R^{2}\right)$ vs $L^{-1}$. Open circles: longer tubes. Filled squares: shorter tubes. Dashed line: fit to data to an interpolation formula described in text. Inset: breakpoint position normalized to $L$ vs $L$ on $\log$ scale. 


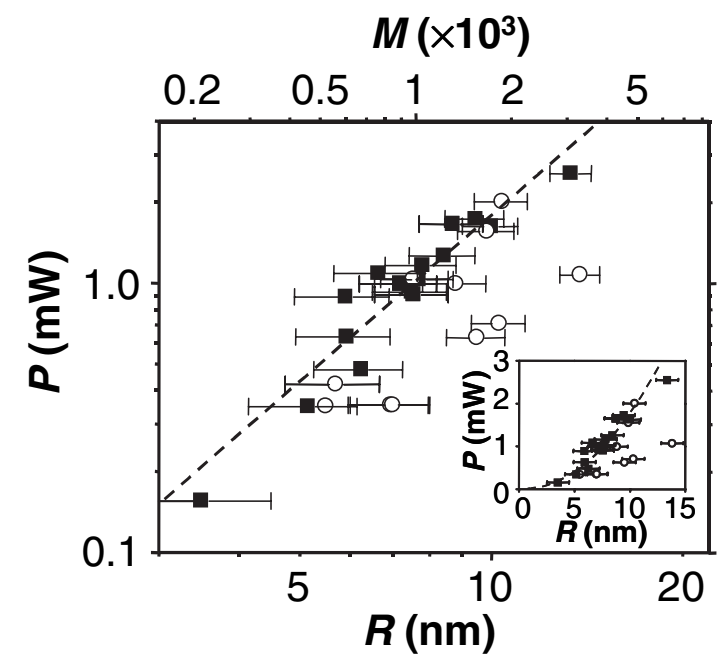

FIG. 3. Log-log plot of $P$ vs $R$ (lower scale) and $M$ (upper scale). Open circles: longer tubes. Filled squares: shorter tubes. Dashed line: power-law fit to short nanotube $P v s R$ and $M$ data. Inset: Linear plot of $P$ vs $R$ and fit.

fore escaping the tube is $\sim L / 2$, we would infer a characteristic umklapp scattering mean-free path $l_{U} \sim 0.2 \mu \mathrm{m}$. We note that this situation, where the electron mean-free path is shorter than $l_{U}$, is reminiscent of the conditions prevailing in silicon-based transistors [22]. This must be accounted for to understand thermal management in $\mathrm{Si}$ transistors. Similar issues may therefore occur in nanotube transistor devices.

In the ballistic regime, the heat flux carried by the phonons $P$ is given by $[3,12]$

$$
P=\sum_{n} \int \frac{d \omega_{n}}{2 \pi} \hbar \omega_{n}\left[\eta_{\mathrm{ne}}\left(\omega_{n}\right)-\eta\left(\omega_{n}, T_{0}\right)\right] \xi\left(\omega_{n}\right)
$$

where the integration is over each of the $n$th photon modes' bandwidths, $\eta_{\text {ne }}\left(\omega_{n}\right)$ is the nonequilibrium phonon distribution for the $n$th phonon branch, $\eta\left(\omega_{n}, T_{0}\right)$ is the BoseEinstein distribution corresponding to the phonons injected into the nanotube at the electrode temperature $T_{0}$, and $\xi\left(\omega_{n}\right)$ is the transmission coefficient for phonons escaping into the electrodes.

We now make several assumptions to simplify Eq. (1). Although the geometry dependence of $\xi\left(\omega_{n}\right)$ for various situations was calculated by Rego and Kirczenow [23], our geometry of an extended lateral contact was not addressed. However, considering the negligibility of $\kappa_{C}$ as discussed above, as well as the relatively large characteristic thermal phonon wave vector $\sim 10^{10} \mathrm{~m}^{-1}$ compared to that studied in Ref. [23], we take $\xi\left(\omega_{n}\right) \approx 1$. Moreover, since $T_{B} \gg T_{0}$ we neglect $\eta\left(\omega_{n}, T_{0}\right)$ relative to $\eta_{\text {ne }}\left(\omega_{n}\right)$. Since breakdown depends on $P$, rather than $V$ or $I$ separately the hot phonons emitted by the electrons likely achieve thermodynamic equilibrium over a thermalization length $l_{\text {th }} \ll L$ after a few $L$-independent characteristic number of collisions [24]. Thus we set $\eta_{\text {ne }}\left(\omega_{n}\right)=\eta\left(\omega_{n}, T\right)$, where $T$ is the tube temperature. We also set $\omega_{n, \min } \approx n c / R$ [25], where $c \sim 1.5 \times 10^{4} \mathrm{~m} / \mathrm{s}$ is the in-plane speed of sound in graphite [26]. Since $T_{B}$ is considerably less than $\Theta_{D} \sim 2500 \mathrm{~K}$, the graphene Debye temperature, we replace $\omega_{n, \max }$ by infinity. Finally, motivated by the observed $P \propto R^{2}$ relationship we assume that the thermal current is carried by the different nanotube shells in parallel.

Summing over the contribution from each shell in the MWNT spaced by $a=0.34 \mathrm{~nm}$ independently, the power dissipated by phonons exiting the nanotube is then

$$
P_{\mathrm{ph}} \approx 2 \zeta(3)\left(k_{B} T\right)^{3} \frac{R^{2}}{\pi \hbar^{2} a c},
$$

taking into account the heat flow into both contacts and phonon mode degeneracy factor of 2 , with $\zeta$ the Riemann zeta function. Note that this expression contains no free parameters. We rewrite Eq. (2) as $P=2 M \kappa_{Q} T$, analogous to the well-known Landauer formula for the ballistic conduction of electrons. Here $M \approx 1.5 \pi k_{B} T R^{2} / h a c$, corresponding to the characteristic number of occupied phonon branches, and $\kappa_{Q}=\pi^{2} k_{B}^{2} T / 3 h$ is the thermal conductance quantum [1,2].

Plotting $P$ vs $M$ for samples shorter than $\sim 0.5 \mu \mathrm{m}$ should thus yield a straight line with a slope of $2 T_{B} \kappa_{Q}$. Figure 3 shows such a plot ( $M$ axis, upper scale) with $T=T_{B}=900 \mathrm{~K}$ for tubes with $L \lesssim 0.5 \mu \mathrm{m}$. The data closely follow a straight line with a fitted slope of $1.0 \mu \mathrm{W} /$ branch. From this, we infer a value for the thermal conductance quantum of $6 \times 10^{-10} \mathrm{~W} / \mathrm{K}$. This is the key experimental finding of this work. Although the accuracy of this measured value of $\kappa_{Q}$ is somewhat limited by the uncertainty in $T_{B}$ and the assumptions of our model, our experimental determination of $\kappa_{Q}$ is nevertheless in good quantitative agreement to the theoretical value $\kappa_{Q}=$ $9 \times 10^{-10} \mathrm{~W} / \mathrm{K}$. This demonstrates that we readily reach quantum mechanical limits to thermal transport in our nanotube devices that act as ballistic phonon waveguides. This is the first such observation for a nanostructure under ambient conditions, enabled by the unique thermal properties of carbon nanotubes.

From our model and the data of Fig. 2, we also obtain an estimate for the phonon mean-free path, which was not determined in Ref. [9]. Since the thermal conductance in the diffusive limit may be written as $K=\lambda M \kappa_{Q} / L$, where $\lambda$ is the phonon mean-free path we can obtain an interpolated expression for the power, appropriate to $T=900 \mathrm{~K}$, that agrees with the asymptotic limits discussed earlier, $P_{\text {int }}=2 M \kappa_{Q} L^{-1} /\left(L^{-1}+3 / 8 \lambda\right)$. The dashed line is a fit of $P_{\text {int }} / 8 \pi R^{2}$ to the data of Fig. 2 with $\lambda=220 \mathrm{~nm}$, yielding a satisfactory fit to the data over the entire length range with only a single free parameter.

Furthermore, our data yield insight into the breakdown process. The well-defined breakdown temperature suggests it requires an initial defect-forming step with activation energy $\Delta$. We expect the defect formation rate is $\Gamma \sim$ $N \omega_{A} \exp \left(-\Delta / k_{B} T_{B}\right)$, where $\omega_{A}$ is an attempt frequency, 


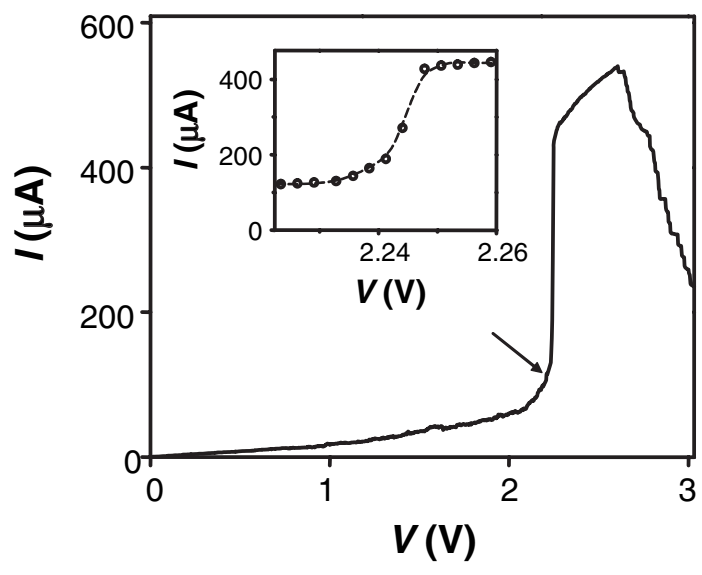

FIG. 4. IV characteristic of a freestanding nanotube device with $R=14 \mathrm{~nm}$. The arrow indicates a dramatic current increase. Inset: expanded view of data in the rapid-increase region.

and $N$ is the number of atoms in the tube at $T_{B}$. We find $\Delta \sim 3-4 \mathrm{eV}$ with $\Gamma=1 \mathrm{~s}^{-1}$, a range of $\omega_{A} \sim$ $10^{8}-10^{18} \mathrm{~s}^{-1}$ and $N \sim 10^{6}$, highly insensitive to the choice of $N$ and $\Gamma$. One possible origin for $\Delta$ is the formation of a Stone-Wales defect. However, our estimated $\Delta$ is considerably smaller than the theoretically determined barrier $\sim 10 \mathrm{eV}$ to form a Stone-Wales defect in graphite and MWNTs [27] ruling out this possible mechanism.

Our estimated $\Delta \sim 3-4 \mathrm{eV}$ is close to the characteristic energy cost $E_{\sigma-\pi^{*}} \sim 3.6 \mathrm{eV}$ (see, e.g., Ref. [26]) of bondweakening $\sigma-\pi^{*}$ electronic transitions. Our data then suggest a shell breakdown mechanism initiated by thermal activation of $\sigma-\pi^{*}$ electronic transitions, similar to the results of scanning tunneling microscope cutting experiments [28] which were interpreted as originating with excitation of such transitions by the tunneling electrons [29].

Finally, we are able to improve nanotube device conductance considerably using the electric current flow. Freestanding samples with initial low-bias resistance $\sim 50 \mathrm{k} \Omega$ up to $10 \mathrm{M} \Omega$ typically show a rapid increase in conductance as the voltage across the sample is ramped, such as shown in Fig. 4. At higher voltages, a cascade of shell ablation begins and the current decreases in a stepwise fashion. The inset shows that the rise in conductance occurs in a smooth fashion. This behavior may be related to the structural annealing recently imaged by TEM [15]. Further experiments, however, are necessary to fully clarify the origins of this behavior, which is of practical value in addressing the challenge of obtaining a high yield of conductive nanotube devices.

We thank Ali Ghaffari for assistance with fabrication. This work was supported by the Office of Naval Research, and Arrowhead Research Corporation (M. B.). The work in Lausanne was supported by the Swiss NSF and its research pool Nanoscale Science (L. F.). C. N. L. acknowledges the support of DARPA/DMEA (H94003-05-2-0504).
*To whom correspondence should be addressed. Email address: mwb@caltech.edu

[1] J. B. Pendry, J. Phys. A 16, 2161 (1983).

[2] R. Maynard and E. Akkermans, Phys. Rev. B 32, 5440 (1985).

[3] K. Schwab et al., Nature (London) 404, 974 (2000).

[4] J. Hone et al., Phys. Rev. B 59, R2514 (1999).

[5] J. Hone et al., Science 289, 1730 (2000).

[6] M. C. Llaguno et al., Nano Lett. 4, 45 (2004).

[7] J.P. Small, K. M. Perez, and P. Kim, Phys. Rev. Lett. 91, 256801 (2003).

[8] P. Kim et al., Phys. Rev. Lett. 87, 215502 (2001).

[9] E. Brown et al., Appl. Phys. Lett. 87, 023107 (2005).

[10] P. G. Collins et al., Phys. Rev. Lett. 86, 3128 (2001).

[11] B. Bourlon et al., Phys. Rev. Lett. 92, 026804 (2004).

[12] T. Yamamoto, S. Watanabe, and K. Watanabe, Phys. Rev. Lett. 92, 075502 (2004).

[13] J. Nygard and D. H. Cobden, Appl. Phys. Lett. 79, 4216 (2001).

[14] M. Bockrath et al., Science 275, 1922 (1997).

[15] J. Y. Huang et al., Phys. Rev. Lett. 94, 236802 (2005).

[16] J. Cumings, P. G. Collins, and A. Zettl, Nature (London) 406, 586 (2000).

[17] We carefully calibrated the SEM radius measurements by comparing SEM images to AFM images on the same nanotube, for a selected subset of the nanotubes. We also note that the typical diameter of the nanotubes used in this study is $\sim 15-25 \mathrm{~nm}$, which exceeds our SEMs typical resolution of $<3 \mathrm{~nm}$.

[18] We note that this cannot be explained by electrical contact resistance. Power dissipation at the contacts should be less effective in heating the nanotube than power dissipation in its bulk, because the contacts provide the most direct thermal anchoring to ambient temperature conditions. This would increase the breakdown power for shorter tubes, contradicting the saturation behavior we observe.

[19] S. Frank et al., Science 280, 1744 (1998).

[20] J. Y. Park et al., Nano Lett. 4, 517 (2004).

[21] Z. Yao, C. Kane, and C. Dekker, Phys. Rev. Lett. 84, 2941 (2000).

[22] D. G. Cahill et al., J. Appl. Phys. 93, 793 (2003).

[23] L. G. C. Rego and G. Kirczenow, Phys. Rev. Lett. 81, 232 (1998).

[24] Additionally, we expect that the typical phonon-phonon scattering mean-free path $l_{p-p}$ should be smaller than the umklapp scattering length by $l_{p-p} \sim l_{U} \exp \left(-\Theta_{D} / T_{B}\right) \sim$ 10-20 nm. Given the relatively few collisions (independent of $L$ ) required for thermalization, we expect on these grounds also that $l_{\text {th }} \ll L$.

[25] L. X. Benedict, S. G. Louie, and M. L. Cohen, Solid State Commun. 100, 177 (1996).

[26] M.S. Dresselhaus, G. Dresselhaus, and P.C. Eklund, Science of Fullerenes and Carbon Nanotubes (Academic Press, New York, 1996).

[27] E. Kaxiras and K. C. Pandey, Phys. Rev. Lett. 61, 2693 (1988).

[28] L. C. Venema et al., Appl. Phys. Lett. 71, 2629 (1997).

[29] A. Rubio et al., Eur. Phys. J. B 17, 301 (2000). 